\title{
Geography and equity: expanding access to obesity medicine diplomate care
}

(c) The Author(s), under exclusive licence to Springer Nature Limited 2021

International Journal of Obesity (2022) 46:447-448;

https://doi.org/10.1038/s41366-021-01044-5

The prevalence of obesity continues to increase worldwide, doubling in over 70 countries between 1980 and 2015 and creating significant burdens of cardiovascular, oncologic, endocrine, and musculoskeletal disease [1]. In the United States (US), efforts to mitigate the health consequences of obesity have included the creation of the American Board of Obesity Medicine (ABOM). Since 2011, ABOM has certified physicians in the United States or Canada as diplomates of obesity medicine through two pathways: completion of a clinical fellowship with a significant obesity component or completion of at least $60 \mathrm{~h}$ of continuing medical education (CME) on obesity, in addition to board examination [2]. Physicians with ABOM certification provide valuable evidence-based care [3]. Yet program success pivots on access: how the growing number of ABOM diplomates are distributed relative to individuals with obesity. A 2019 analysis demonstrated U.S. state variation among 2577 ABOM diplomates; the number of adults with obesity per diplomate ranged from 18,529 (Delaware) to 104,442 (South Dakota), demonstrating regional disparities and overall insufficiency of specialists [4]. In this issue, Pollack and colleagues reveal the most comprehensive longitudinal study to date of $\mathrm{ABOM}$ diplomate availability in the United States. They further our understanding of geographic access and equity in obesity medicine [5].

Pollack and colleagues compare access from 2011 to 2019 based on average travel time from the population-weighted center of each Census tract to the nearest ABOM diplomate in a non-pediatric specialty. Consistent with prior studies, the authors demonstrate very limited accessibility in 2011, estimating over $30 \%$ of American adults with obesity resided over one hour from an obesity medicine diplomate. As the number of $A B O M$ diplomates grew, the estimated average travel time to an obesity medicine specialist fell from $28.5 \mathrm{~min}$ (2011) to $10.0 \mathrm{~min}$ (2019). Reassuringly, analysis by race/ethnicity, household income, rurality, region, and other sociodemographic characteristics revealed improved travel times across examined subgroups.

The authors estimate provider capacity to meet potential demand based on the regional prevalence of obesity, assumed patient time per provider, and visit frequency as suggested by three guidelines. By the most generous assessment, fewer than 1 in 50 facilities in 2019 had adequate specialist capacity to meet the needs of all patients with obesity (defined as BMI $\geq 30 \mathrm{~kg} / \mathrm{m}^{2}$ ), and fewer than 1 in 11 to meet only the needs of patients with severe obesity (BMI $\geq 40 \mathrm{~kg} / \mathrm{m}^{2}$ ).

These estimates come with caveats. ABOM diplomates were assumed to stay at the facility where they were certified; assumed to dedicate a major portion of their clinical practice to obesity medicine, almost certainly an overestimate; and analyzed relative to regional patient data lacking individual granularity. Nonetheless, Pollack and colleagues contribute a valuable snapshot of ABOM diplomate availability in the United States - how far we have come since 2011, and how far we have yet to go. We highlight barriers and opportunities to expand and improve obesity medicine care through undergraduate and graduate medical education, research, professional organizations, and legislative advocacy.

\section{EDUCATION}

Worldwide, medical schools and residencies include minimal obesity medicine training [6]. To integrate comprehensive obesity medicine curricula at an early stage would equip the broadest possible provider population with core competencies to treat obesity. For physicians who elect for additional training, the number of obesity medicine fellowship programs is growing (from 11 to 17 adult programs in 2021 alone) but most programs support only one fellow per year. The ABOM designation of diplomate-and analyses that use it-conflate fellowship-trained and CME-trained obesity medicine specialists. Both are important avenues to expand the field. The fellowship pathway may be more likely to produce specialists who will dedicate their practice to care of patients with obesity.

\section{RESEARCH}

The gap between current provider bandwidth and potential demand for obesity specialist care invites the question: How do we identify the patients most likely to benefit from obesity specialist care, including behavioral and nutritional counseling, anti-obesity medications, and bariatric surgery/endoscopy? New tools to identify high-risk patients (e.g., biomarkers and elastography for early detection of fatty liver disease) can help triage patients. Longitudinal cohort data such as the recent SPLENDOR study of bariatric surgery in people with obesity and nonalcoholic steatohepatitis enable us to quantify benefits and risks of anti-obesity interventions for specific populations [7]. Research must also keep an eye toward equity. For example, we must interpret cautiously the shorter average travel times Pollack and colleagues describe for Black, Asian, Pacific Islander, and Hispanic groups relative to White and Non-Hispanic groups; distrust of medical care, particularly among groups historically marginalized in the healthcare system, financial or childcare demands, inadequate public transportation, and higher costs of city living are not reflected in analyses of geographic proximity to healthcare. 


\section{PROFESSIONAL ORGANIZATIONS}

Obesity medicine groups have a key role in enabling access and uptake of obesity specialist care. Pollack and colleagues show improved average travel times and geographic access to adult obesity specialist care. However, specialist appointment wait times are often several months. Since 2018, the Obesity Medicine Association (OMA) has offered the NP/PA Certificate of Advanced Education in Obesity Medicine to advanced practice providers (APPs) who are ineligible for the ABOM examination. Professional organizations can augment availability through APP training pathways as appointment wait times are typically shorter for APPs [8]. Professional organizations are also well-positioned to amplify high-value treatments through provider-targeted guidelines and public-targeted media campaigns, combating limited knowledge of obesity treatment options. The European Association for the Study of Obesity (EASO) is a federation of obesity associations across 36 countries; its clinical practice guidelines are one model for distillation and distribution of knowledge. Finally, professional organizations are key stakeholders to address racial, ethnic, and socioeconomic disparities in the prevalence and treatment of obesity [9]. Targeted outreach to communities most affected by obesity and efforts to build diverse representation within membership of obesity professional associations help us deliver equitable care.

\section{LEGISLATIVE ADVOCACY}

In many countries, incomplete and variable financial coverage for pharmacotherapy and bariatric surgery deter patients and providers [10]. Obesity specialists can advocate for legislation, like the Treat and Reduce Obesity Act (TROA) in the US, to expand public health insurance coverage for these evidence-based treatments. Similarly, telemedicine is well-suited to reach rural or socially isolated patients; legislation to ensure payment equal to in-person service strengthens incentives to improve geographic access.

Pollack and colleagues characterize incremental progress in obesity specialist availability in the United States. The fivefold growth of ABOM diplomate membership from 2011 to 2019 is worthy of celebration. But this work also highlights significant gaps in universal access to evidence-based specialist care for obesityserving as an impetus to expand the field of obesity medicine and a reminder to address multiple dimensions of access to care.

$$
\begin{array}{r}
\text { Matthew J. Townsend (iD) }{ }^{1} \text {, Niyoti Reddy } \text { (DD }^{2} \text { and } \\
\text { Fatima C. Stanford (iD) }
\end{array}
$$

${ }^{7}$ Duke University Medical Center, Durham, NC, USA. ${ }^{2}$ Massachusetts General Hospital, Boston, MA, USA. ${ }^{3}$ Harvard Medical School, Boston, MA, USA. ${ }^{\circledR}$ email: fstanford@mgh.harvard.edu

\section{REFERENCES}

1. GBDO Collaborators, Afshin A, Forouzanfar MH, Reitsma MB, Sur P, Estep K, et al. Health effects of overweight and obesity in 195 countries over 25 years. N Engl J Med. 2017;377:13-27.
2. Why Certify?: American Board of Obesity Medicine. 2021. https://www.abom.org/\#.

3. Gudzune KA, Wickham EP 3rd, Schmidt SL, Stanford FC. Physicians certified by the American Board of Obesity Medicine provide evidence-based care. Clin Obes. 2021:11:e12407.

4. Gudzune KA, Johnson VR, Bramante CT, Stanford FC. Geographic availability of physicians certified by the American Board of Obesity Medicine relative to obesity prevalence. Obesity. 2019;27:1958-66.

5. Pollack CC, Onega T, Emond JA, Vosoughi S, Malley AJO, McClure AC, et al. A national evaluation of geographic accessibility and provider availability of obesity medicine diplomates in the United States between 2011 and 2019. Int J Obes. 2021.

6. Mastrocola MR, Roque SS, Benning LV, Stanford FC. Obesity education in medical schools, residencies, and fellowships throughout the world: a systematic review. Int J Obes. 2020;44:269-79.

7. Aminian A, Al-Kurd A, Wilson R, Bena J, Fayazzadeh H, Singh T, et al. Association of bariatric surgery with major adverse liver and cardiovascular outcomes in patients with biopsy-proven nonalcoholic steatohepatitis. JAMA. 2021;326:2031-42. https://doi.org/10.1001/jama.2021.19569.

8. Alexander L. The benefits of obesity medicine certification. Am J Lifestyle Med. 2019;13:161-4.

9. Byrd AS, Toth AT, Stanford FC. Racial disparities in obesity treatment. Curr Obes Rep. 2018;7:130-8.

10. Uerlich MF, Yumuk V, Finer N, Basdevant A, Visscher TL. Obesity management in Europe: current status and objectives for the future. Obes Facts. 2016;9:273-83.

\section{AUTHOR CONTRIBUTIONS}

MJT contributed to the concept, design, drafting, and revision of the manuscript. NR contributed critical revision of the manuscript for important intellectual content. FCS contributed supervision, data interpretation, and concept and critical revision of the manuscript for important intellectual content.

\section{FUNDING}

National Institutes of Health NIDDK P30 DK040561 (FCS) and L30 DK118710 (FCS).

\section{COMPETING INTERESTS}

The authors declare no competing interests.

\section{ADDITIONAL INFORMATION}

Correspondence and requests for materials should be addressed to Fatima $C$. Stanford.

Reprints and permission information is available at http://www.nature.com/ reprints

Publisher's note Springer Nature remains neutral with regard to jurisdictional claims in published maps and institutional affiliations. 